\title{
Correlation between habituation of visual-evoked potentials and magnetophosphene thresholds in migraine: A case-control study
}

Cephalalgia

2016, Vol. 36(3) 258-264

(C) International Headache Society 2015 Reprints and permissions: sagepub.co.uk/journalsPermissions.nav DOI: |0.1 |77/03331024|559024| cep.sagepub.com

@SAGE

\author{
Anna Ambrosini', Ennio lezzi ${ }^{2}$, Armando Perrotta', \\ Aliaksei Kisialiou ${ }^{3}$, Andrea Nardella ${ }^{2}$, Alfredo Berardelli ${ }^{2,4}$, \\ Francesco Pierelli ${ }^{1,5}$ and Jean Schoenen ${ }^{6}$
}

\begin{abstract}
Introduction: In migraine most studies report an interictal deficit of habituation of visual-evoked potentials (VEP-hab) and reduced thresholds for phosphene induction (PT) by transcranial magnetic stimulation (TMS). We searched for a possible correlation between VEP-hab and PT in migraine patients and healthy controls to test whether they reflect the same pathophysiological abnormality.

Methods: We assessed PT and VEP-hab measured as the percentage change of NI/PI amplitude over six blocks of 100 responses in 15 healthy volunteers (HV) and in 13 episodic migraineurs without aura (MO) between attacks. Results were compared using Mann-Whitney $U$ test. Interrelationships were examined using Spearman's correlation.

Results: In MO patients VEP-hab was reduced compared to HV $(p=0.00 \mathrm{I})$, while PT were not significantly different between HV and MO. There was no correlation between PT and VEP-hab in either group of participants.

Conclusions: We confirm that in interictal migraine VEP habituation is deficient, but magnetophosphene threshold normal. VEP-hab and PT were not correlated with each other in healthy controls or in migraineurs. This finding suggests that they index different facets of cortical excitability in migraine, i.e. a punctual normal measure of the cortical activation threshold for PT and a dynamic response pattern to repeated stimuli for VEP habituation.
\end{abstract}

\section{Keywords}

Migraine, cortical excitability, magnetophosphene threshold, visual-evoked potentials, habituation

Date received: 27 November 20I4; revised: 2 March 20I5; 2 April 20I5; 16 April 20I5; accepted: 25 April 2015

\section{Introduction}

Migraine is one of the most common neurological disorders, with a high prevalence in the general population (1), and is the result of genetic, environmental and endogenous cognitive-emotional factors (2). The resulting "migraine susceptibility" differs among individuals and fluctuates in the same person over the so-called "migraine cycle." Since interictal symptoms or brain lesions are absent in its episodic form (3), migraine is commonly considered a functional brain disorder. The brain structures involved in the pathophysiology of migraine include the cerebral cortex, the brainstem, the hypothalamus, the thalamus and the trigeminovascular system. The exact sequence of activation of these structures before, during, and after a migraine attack is still unknown $(2,4)$.
Electrophysiological methods are particularly suited to study the functional brain changes associated with

\footnotetext{
'IRCCS Neuromed, Headache Unit, Italy

${ }^{2}$ IRCCS Neuromed, Clinical Neurophysiology Unit, Italy

${ }^{3}$ IRCCS San Raffaele Pisana, Unit of Clinical and Molecular Epidemiology, Italy

${ }^{4}$ Sapienza University of Rome, Department of Neurology and Psychiatry, Italy

${ }^{5}$ Sapienza University of Rome, Department of Medical-Surgical Sciences and Biotechnologies, Italy

${ }^{6}$ University of Liège - CHR Citadelle, University Dept. of Neurology,

Headache Research Unit, Belgium
}

\section{Corresponding author:}

Anna Ambrosini, Headache Unit - IRCCS Neuromed, via Atinense, 18 I-86077, Pozzilli (Isernia), Italy.

Email: anna.ambrosini@neuromed.it 
migraine (5). A reduced habituation of cortical-evoked responses to repeated stimuli was the first reproducible physiological brain abnormality found interictally in migraine patients (6). It has been described for almost every stimulation modality (visual, auditory, somatosensory, olfactory, nociceptive) and for event-related potentials such as contingent negative variation and P300. In healthy individuals, repetitive stimuli are associated with a progressive decrease in amplitude of cortical-evoked responses, a phenomenon called "habituation" that is defined as "a response decrement as a result of repeated stimulation" (7). Migraine patients may have interictally a reduced habituation or even an amplitude increase ("potentiation"). The majority of studies on habituation in migraineurs were performed with visual stimuli using pattern-reversal visual-evoked potentials (VEP-hab) (8). VEP abnormalities, however, have been reported in most but not in all studies $(8,9)$, and differences between results have been explained on the basis of methodological differences between the various studies, fluctuations of cortical information processing over the migraine cycle, and/or differences in patient populations regarding attack frequency or genetic factors (8). One important methodological difference is whether blinding for diagnosis during VEP recordings and analyses was used (9-11).

Another abnormality previously reported in migraine patients - particularly in patients with aura - is an increased prevalence $(12,13)$ and a lower threshold of phosphenes (PT) induced by transcranial magnetic stimulation (TMS) of the occipital cortex, which is generally thought to reflect hyperexcitability of the visual cortex (12,14-22). Conversely, several other studies found no differences in PT between migraineurs and controls (23-26).

Both VEP-hab deficit and reduced PT are commonly considered as an expression of increased excitability of cortical areas (e.g. Haigh et al. (27); Mickleborough et al. (28); Brennan (29); Lang et al. (30); and Lipton and Pearlman (31)). However, though an increased excitability may explain PT changes, this may not be true for VEP-hab changes, because, if it were, one would expect to find increased VEP amplitude for a low number of stimuli, which is not the case (32). Given these uncertainties, important additional information would be to verify if there is an intra-individual correlation between VEP-hab and PT in normal conditions and in migraine patients. If there were a correlation between VEP-hab and PT, one might conclude that a similar neurophysiological mechanism explains the abnormalities found with the two techniques (27-31). To this aim we explored PTs and VEP-hab in the same recording session in a group of episodic migraineurs without aura in the interictal phase, searching for correlations between PT values and VEP-hab measures, and comparing these results with those obtained from a group of healthy controls investigated in similar conditions.

\section{Methods}

\section{Participants}

Thirteen patients suffering from episodic migraine without aura (MO, (ICHD-3 beta) 1.1) were recruited from the outpatient Headache Clinic of the Neuromed Institute (Pozzilli, Italy) and were compared to a group of 15 healthy volunteers (HVs) with no familial history of recurrent headaches. An "a posteriori" sample size calculation, assuming type I error $\alpha$ (significance) $=0.05$ and type II error $\beta$ (1-power $)=0.20$, showed that our sample size had an $80 \%$ power to detect the correlation between VEP-hab and PTs.

Demographic and clinical details of both groups are summarized in Table 1. None of the participants had an uncorrected visual deficit, was taking drugs on a regular basis, or had taken any drug within three days before the recordings. The recordings took place at least three days after or before an attack; the occurrence of attacks after the recording was verified by a telephone call.

The study was conducted after approval by our institution's ethics committee, in accordance with the ethical standards of the 1964 Declaration of Helsinki, with the understanding and informed written consent of each involved individual.

\section{Experimental procedures}

VEP recordings and PT measures were performed on the same day (in a pseudo-randomized order at least 30 minutes apart) by E.I and A.N., who were both blinded to diagnoses. Patients and healthy volunteers were instructed not to mention their respective condition to the investigators. All experiments were performed at a comparable time of day.

VEPs. Participants were comfortably seated in a dark room in front of a television monitor (16.5 inches, mean luminance $260 \mathrm{~cd} / \mathrm{m}^{2}$ ) placed $100 \mathrm{~cm}$ from their eyes and connected to a Medelec Synergy, PLINTH, CareFusion (Middleton, WI, USA) device. Stimuli were presented as a checkerboard pattern of black and white squares (contrast $80 \%$ ), subtending one degree, eight minutes of arc, at a reversal rate of $3.1 \mathrm{~Hz}$. With one eye patched, individuals were instructed to fix on a red dot placed in the middle of the screen. Recordings were performed after at least one minute of adaptation to darkness. Needle electrodes were positioned at $\mathrm{Oz}$ (active electrode) and at $\mathrm{Fz}$ (reference) according to the 10-20 
Table I. Demographic and clinical characteristics.

\begin{tabular}{|c|c|c|c|c|c|c|c|c|}
\hline & $N$ & Gender & Male \% & Age (SD), years & $\begin{array}{l}\text { Mean headache } \\
\text { frequency (SD) } \\
\text { (monthly) }\end{array}$ & $\begin{array}{l}\text { Mean headache } \\
\text { days (SD) } \\
\text { (monthly) }\end{array}$ & $\begin{array}{l}\text { Mean headache } \\
\text { duration (SD) } \\
\text { (hours) }\end{array}$ & $\begin{array}{l}\text { Disease } \\
\text { duration (SD) } \\
\text { (years) }\end{array}$ \\
\hline HVs & 15 & $8 \mathrm{~F} / 7 \mathrm{M}$ & $46.7 \%$ & $\begin{array}{l}30.0(7.9) \\
\quad(\text { range } 21-44)\end{array}$ & I & I & I & I \\
\hline MO & 13 & $7 \mathrm{~F} / 6 \mathrm{M}$ & $46.2 \%$ & $\begin{array}{l}32.8(10.2) \\
\quad(\text { range 18-55) }\end{array}$ & $3.2(1.5)$ & $4.2(2.7)$ & $10.4(5.6)$ & II.7(6.8) \\
\hline
\end{tabular}

HVs: healthy volunteers; MO: migraineurs without aura; F: female; M: male.

electroencephalogram (EEG) system. The ground electrode was placed on the forearm. During repetitive stimulation, six consecutive blocks of 100 responses were recorded (sweep duration: $300 \mathrm{msec}$, bandpass: $1-100 \mathrm{~Hz}$ ) and on-line averaged. The six blocks were analyzed in terms of peak-to-peak amplitudes of the N1-P1 components identified by visual inspection. The N1/P1 amplitudes in successive blocks were measured and VEP habituation was expressed as the percentage change of N1/P1 amplitude between the sixth and the first blocks (VEP-hab percentage: negative sign = habituation, positive sign = potentiation) and as habituation slopes (linear regression line amplitude $/ N$ blocks) (VEP-hab slopes: negative sign = habituation, positive sign $=$ potentiation). Two investigators (E.I. and A.N.) blinded to the diagnosis analyzed the VEPs.

Magnetophosphene thresholds. The participants, while sitting comfortably in an armchair in a dark room, were blindfolded and wore earplugs. After at least one minute of adaptation to the dark, TMS was performed with a MAGSTIM SuperRapid stimulator delivering biphasic waveform pulses (The MAGSTIM Company Ltd, Whitland, Dyfed, UK). The stimulator was connected to a standard figure-of-eight coil (outer diameter: $9 \mathrm{~cm}$ ) held in a vertical position (handle of the coil pointing upward, coil placed tangentially to the scalp). The induced current flowed in the coil with a clockwise-counter clockwise direction. Paired stimuli (19) (with an interstimulus interval of $50 \mathrm{~ms}$ ) were used. The individuals' visual cortex was identified by positioning the coil $3-4 \mathrm{~cm}$ above the inion and delivering paired stimuli at $70 \%$ of maximum stimulator output (MSO), thereafter increasing strength by $5 \%$ steps. If no phosphenes were obtained, the same procedure was repeated $1 \mathrm{~cm}$ up, down, or to the right or left, until phosphenes occurred. Once the hotspot was identified, the PT was measured starting with a stimulus intensity of $40 \%$ MSO with increasing/decreasing $5 \%$ steps and fine-tuning of $1-2 \%$. To determine PTs, 15.2 (standard deviation (SD) 5.6) pairs of stimuli were necessary. In order to avoid possible effects on cortical excitability due to repeated stimulation, paired stimuli were delivered with variable intervals of at least five seconds.

\section{Statistical analyses}

Results were expressed as means and SD for quantitative variables. Between-group differences in demographics, VEP-hab percentages, VEP-hab slopes, and PTs were tested by the Mann-Whitney $U$ test. Intra-individual correlations between PT and VEP-hab percentages, VEP-hab slopes, and first block VEP amplitude were determined in both HV and MO groups by Spearman's rho non-parametric correlation test. Significance level was set at $p \leq 0.05$. Statistical calculations were carried out using IBM Corp. released 2010 (IBM SPSS Statistics for Windows, version 19.0. Armonk, NY, USA, and $R$ version 3.0.2. for Windows).

\section{Results}

\section{Group comparisons}

Age and gender distributions were not significantly different between HV and MO groups. Mean VEP-hab percentage was clearly abnormal in MO, who had potentiation $(+23.6 \%$ SD 20.5$)$ compared to $\mathrm{HVs}$ who had habituation $(-19.2 \%$ SD 15.5$)(p=0.001)$. VEP-hab slopes were significantly steeper in the HV group $(-0.340 \mu \mathrm{V} / \mathrm{N}$ SD 0.250$)$ than in the $\mathrm{MO}$ $(+0.276 \mu \mathrm{V} / \mathrm{N}$ SD 0.246) $(p=0.001)$. First block VEP amplitude was lower in MO $(8.51 \mu \mathrm{V}$ SD 3.26) than in HVs (10.64 $\mu$ V SD 2.99), but this did not reach the level of significance $(p=0.134)$. All participants experienced phosphenes during TMS over the visual cortex and hence a $100 \%$ prevalence was found both in MO and HVs. Similarly, PT were not significantly different between MO (65.46\% SD 14.01) and HV groups (58.6\% $\mu \mathrm{V}$ SD 6.39) $(p=0.249)$.

\section{Intraindividual correlations}

No correlation was found in the HV group between PT and VEP-hab percentages (Spearman's rho $=0.090$ ), 


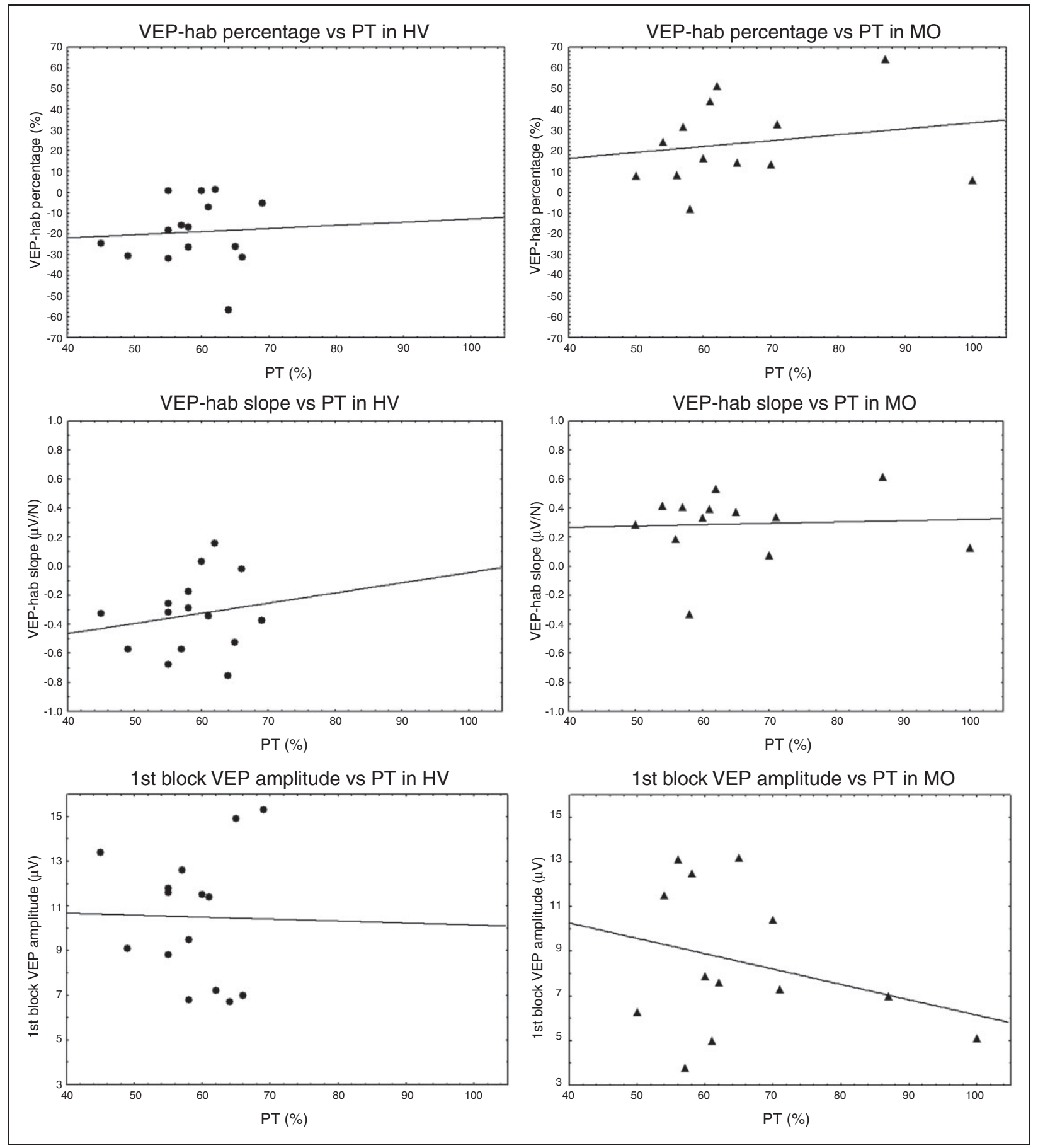

Figure I. Scatterplots of correlations between phosphene thresholds and VEP-hab percentages (higher part of the picture), VEP-hab slopes (medium part of the picture) and first block VEP amplitude (lower part of the picture) both for healthy volunteers (HV: circles) and migraineurs without aura (MO: triangles). The black lines express the related linear regression lines. VEP-hab: interictal deficit of habituation of visual-evoked potentials.

between PT and VEP-hab slopes (Spearman's rho $=0.140$ ) or between PT and first block VEP amplitudes (Spearman's rho $=-0.091$ ). MO patients also disclosed no correlation between PT and VEP-hab percentages (Spearman's rho $=0.253$ ), PT and VEPhab slopes (Spearman's rho $=0.033$ ) or PT and first block VEP amplitudes (Spearman's rho $=-0.154$ ) (Figure 1). 


\section{Discussion}

In this study, we investigated whether in migraine patients and healthy subjects magnetophosphene thresholds (PTs) and VEP habituation/first block amplitudes correlate. Studying the same patients with both techniques and in the same experimental session, a correlation between PTs and VEP habituation would suggest that PTs and VEP-hab would share the same pathophysiological mechanisms.

In our study, where recordings and analyses were performed blindly, we found an interictal deficit of VEP habituation in patients suffering from episodic migraine, in line with the majority of VEP studies in migraine, most of which were unblinded (8). This finding further confirms that blinding may be useful but not necessary when exploring evoked potentials habituation in migraine. In agreement with other studies (32), there was a trend for lower amplitude of the first VEP block in migraine. Finally, PTs in migraine patients were not different from those of normal participants. This finding was similar to that reported by several authors (23-26) but at variance with several others $(15,17,19-22)$, we found no significant decrease of PT in migraineurs.

The habituation deficit could simply be due to cortical hyperexcitability. This is not, however, supported by the previous finding that amplitude of the first VEP block is rather low in comparison to normal individuals (32). Based on the so-called "ceiling theory" of cortical activation (33), it has been proposed that the habituation deficit and the reduced VEP amplitude of the first block could be a consequence of reduced pre-activation levels of sensory cortices. The very first responses to peripheral stimuli in low pre-activated sensory cortices would be small sized, but, as cortical activation rises to its maximum ("ceiling") during repetitive stimulation, their amplitudes would progressively increase, which is what actually happens in evoked potentials elicited in migraine patients. The finding that excitatory highfrequency TMS is able to enhance VEP habituation in migraineurs (34) further supports the hypothesis that the cortical pre-activation level in migraineurs is reduced. Following this interpretation, the cerebral cortex would thus be "hyperresponsive" in migraine between attacks rather than "hyperexcitable" $(35,36)$.

Studies with TMS of the occipital cortex have reported increased phosphene prevalence and/or thresholds particularly in migraine with aura $(12,14$ 22 ), suggesting therefore an increased excitability of cerebral cortex. By contrast, several authors reported opposite results (23-26): lower phosphene prevalence in MA but no difference between controls and MO patients, and similar mean PT in individuals reporting phosphenes, which was confirmed in the present investigation. In a study measuring PT over a 10-week period, Antal et al. (23) found no significant difference between migraineurs and HVs; however, they noticed a high variability of PT over time in migraine patients, favoring instability of cortical excitability instead of hyperexcitability.

If changes in PT and VEP habituation would simply reflect cortical hyperexcitability, one would expect to find an inverse relationship between PT and VEP habituation or first block amplitudes, both being directly correlated (32). Our finding that neither in HVs nor in migraine patients PT and VEP habituation/first block amplitudes are correlated suggests that they index different functional properties of the visual cortex. VEP habituation most likely represents a dynamic response pattern of sensory cortices to repeated stimuli, providing a temporal profile of cortical reactions to incoming inputs, whereas PT indicates purely a measure of cortical activation when the information reaches the cortex. Magnetophosphenes likely reflect the activation of visual cortical cells, but whether striate or extrastriate cortices and/or geniculostriate fibers are involved in their generation is still unclear (37). Studies combining EEG and TMS techniques suggest that the perceptual awareness of phosphenes elicited by TMS is not an immediate consequence of visual cortices activation and that many other cortical areas, such as parietal and temporal cortices, are involved in phosphene perception $(38,39)$. One combined VEP and functional magnetic resonance imaging (fMRI) study, however, suggested that N1-P1 components of pattern-reversal VEPs are most likely generated in the striate cortex V1 (40), although other studies with magnetoencephalographic (MEG) analyses (41) and combined MEGfMRI investigations suggested an activation of both striate (V1) and extrastriate cortices (V2) (42). Different neuroanatomical substrates may thus explain the lack of correlation we found between VEP-hab and PTs.

In conclusion, the absence of correlation between VEP-hab and PT both in healthy subjects and in migraine patients provides evidence that they do not index the same cortical mechanisms. Moreover, our study is yet another confirmation that VEPs are better suited than PT to detect an abnormal function of the visual cortex in migraineurs and that this abnormality is not simple hyperexcitability, but it represents more likely a dynamic increase of cortical responsivity to repeated visual stimulation. 


\section{Article highlights}

- In this blinded study of visual-evoked potentials (VEPs), we confirm that migraine without aura patients have an interictal deficit of habituation to repetitive visual stimuli.

- Magnetophosphene thresholds (PTs) did not differ between migraineurs and healthy volunteers.

- There was no intraindividual correlation between VEP habituation values and PTs in either migraine patients or in healthy controls, suggesting that the two tests do not reflect the same cortical property.

- Our findings favor the concept that in migraine between attacks the visual cortex is not hyperexcitable, but hyperresponsive.

\section{Funding}

This research received no specific grant from any funding agency in the public, commercial, or no-for-profit sectors.

\section{Conflict of interest}

None declared.

\section{References}

1. Stovner LJ and Andrée C. Prevalence of headache in Europe: A review for the Eurolight project. $J$ Headache Pain 2010; 11: 289-299.

2. Goadsby PJ, Charbit AR, Andreou AP, et al. Neurobiology of migraine. Neuroscience 2009; 161: 327-341.

3. Headache Classification Subcommittee of the International Headache Society. The International Classification of Headache Disorders, 3rd edition (beta version). Cephalalgia 2013; 33: 629-808.

4. Bernstein $\mathrm{C}$ and Burstein R. Sensitization of the trigeminovascular pathway: Perspective and implications to migraine pathophysiology. J Clin Neurol 2012; 8: 89-99.

5. Magis D, Ambrosini A, Bendtsen L, et al. Evaluation and proposal for optimalization of neurophysiological tests in migraine: Part 1-electrophysiological tests. Cephalalgia 2007; 27: 1323-1338.

6. Schoenen J, Wang W, Albert A, et al. Potentiation instead of habituation characterizes visual evoked potentials in migraine patients between attacks. Eur $J$ Neurol 1995; 2: 115-122.

7. Harris J. Habituatory response decrement in the intact organism. Psychol Bull 1943; 40: 385-422.

8. De Tommaso M, Ambrosini A, Brighina F, et al. Altered sensory processing in migraine. Nat Rev Neurol 2014; 10 : 144-155.

9. Omland PM, Nilsen KB, Uglem M, et al. Visual evoked potentials in interictal migraine: No confirmation of abnormal habituation. Headache 2013; 53: 1071-1086.

10. Bednář M, Kubová Z and Kremláček J. Lack of visual evoked potentials amplitude decrement during prolonged reversal and motion stimulation in migraineurs. Clin Neurophys 2014; 125: 1223-1230.

11. Ambrosini A, Schoenen J, Magis D, et al. EHMTI-0222. Habituation of visual evoked potentials in migraine:
Comparison between blinded and non-blinded analyses. J Headache Pain 2014; 15(Suppl 1): E1.

12. Aurora SK, Ahmad BK, Welch KMA, et al. Transcranial magnetic stimulation confirms hyperexcitability of visual cortex in migraine. Neurology 1998; 50: 1105-1110.

13. Brighina F, Piazza A, Daniele O, et al. Modulation of visual cortical excitability in migraine with aura: Effects of $1 \mathrm{~Hz}$ repetitive transcranial magnetic stimulation. Exp Brain Res 2002; 145: 177-181.

14. Aurora SK, Cao Y, Bowyer SM, et al. The occipital cortex is hyperexcitable in migraine: Experimental evidence. Headache 1999; 39: 469-476.

15. Mulleners WM, Chronicle EP, Palmer JE, et al. Visual cortex excitability in migraine with and without aura. Headache 2001; 41: 565-572.

16. Aurora SK, Welch KM and Al-Sayed F. The threshold for phosphenes is lower in migraine. Cephalalgia 2003; 23 : 258-263.

17. Young WB, Oshinsky ML, Shechter AL, et al. Consecutive transcranial magnetic stimulation: Phosphene thresholds in migraineurs and controls. Headache 2004; 44: 131-135.

18. Aurora SK, Barrodale P, Chronicle EP, et al. Cortical inhibition is reduced in chronic and episodic migraine and demonstrates a spectrum of illness. Headache 2005; 45: 546-552.

19. Gerwig M, Niehaus L, Kastrup O, et al. Visual cortex excitability in migraine evaluated by single and paired magnetic stimuli. Headache 2005; 45: 1394-1399.

20. Khedr EM, Ahmed MA and Mohamed KA. Motor and visual cortical excitability in migraineurs patients with or without aura: Transcranial magnetic stimulation. Neurophysiol Clin 2006; 36: 13-18.

21. Gunaydin S, Soysal A, Atay T, et al. Motor and occipital cortex excitability in migraine patients. Can J Neurol Sci 2006; 33: 63-67.

22. Siniatchkin M, Reich AL, Shepherd AJ, et al. Peri-ictal changes of cortical excitability in children suffering from migraine without aura. Pain 2009; 147: 132-140.

23. Antal A, Arlt S, Nitsche MA, et al. Higher variability of phosphene thresholds in migraineurs than in controls: A consecutive transcranial magnetic stimulation study. Cephalalgia 2006; 26: 865-870.

24. Bohotin V, Fumal A, Vandenheede M, et al. Excitability of visual V1-V2 and motor cortices to single transcranial 
magnetic stimuli in migraine: A reappraisal using a figure-of-eight coil. Cephalalgia 2003; 23: 264-270.

25. Brighina F, Piazza A, Daniele O, et al. Modulation of visual cortical excitability in migraine with aura: Effects of $1 \mathrm{~Hz}$ repetitive transcranial magnetic stimulation. Exp Brain Res 2002; 145: 177-181.

26. Afra J, Mascia A, Gérard P, et al. Interictal cortical excitability in migraine: A study using transcranial magnetic stimulation of motor and visual cortices. Ann Neurol 1998; 44: 209-215.

27. Haigh S, Karanovic O, Wilkinson F, et al. Cortical hyperexcitability in migraine and aversion to patterns. Cephalalgia 2012; 32: 236-240.

28. Mickleborough MJ, Hayward J, Chapman C, et al. Reflexive attentional orienting in migraineurs: The behavioral implications of hyperexcitable visual cortex. Cephalalgia 2011; 31: 1642-1651.

29. Brennan KC. Turn down the lights! An irritable occipital cortex in migraine without aura. Neurology 2011; 76: 206-207.

30. Lang E, Kaltenhäuser M, Neundörfer B, et al. Hyperexcitability of the primary somatosensory cortex in migraine - a magnetoencephalographic study. Brain 2004; 127 : 2459-2469.

31. Lipton RB and Pearlman SH. Transcranial magnetic simulation in the treatment of migraine. Neurotherapeutics 2010; 7: 204-212.

32. Coppola G, Currà A, Sava SL, et al. Changes in visualevoked potential habituation induced by hyperventilation in migraine. $J$ Headache Pain 2010; 11: 497-503.

33. Knott JR and Irwin DA. Anxiety, stress and the contingent negative variation. Arch Gen Psychiatry 1973; 29: 538-541.
34. Bohotin V, Fumal A, Bohotin CR, et al. Repetitive transcranial magnetic stimulation of the occipital cortex modifies habituation of visual evoked potentials in healthy volunteers and migraineurs. Cephalalgia 2001; 21: 388-389.

35. Coppola G, Pierelli F and Schoenen J. Is the cerebral cortex hyperexcitable or hyperresponsive in migraine? Cephalalgia 2007; 27: 1427-1439.

36. Coppola G, Ambrosini A, Di Clemente L, et al. Interictal abnormalities of gamma band activity in visual evoked responses in migraine: An indication of thalamocortical dysrhythmia? Cephalalgia 2007; 27: 1360-1367.

37. Merabet LB, Theoret H and Pascual-Leone A. Transcranial magnetic stimulation as an investigative tool in the study of visual function. Optom Vis Sci 2003; 80: 356-368.

38. Taylor PC, Walsh V and Eimer M. The neural signature of phosphene perception. Hum Brain Mapp 2010; 31: 1408-1417.

39. Bagattini C, Mazzi C and Savazzi S. Waves of awareness for occipital and parietal phosphenes perception. Neuropsychologia 2015; 70: 114-125.

40. Di Russo F, Pitzalis S, Spitoni G, et al. Identification of the neural sources of the pattern-reversal VEP. Neuroimage 2005; 24: 874-886.

41. Barnikol UB, Amunts K, Dammers J, et al. Pattern reversal visual evoked responses of $\mathrm{V} 1 / \mathrm{V} 2$ and $\mathrm{V} 5 / \mathrm{MT}$ as revealed by MEG combined with probabilistic cytoarchitectonic maps. Neuroimage 2006; 31: 86-108.

42. Perfetti B, Franciotti R, Della Penna S, et al. Low- and high-frequency evoked responses following pattern reversal stimuli: A MEG study supported by fMRI constraint. Neuroimage 2007; 35: 1152-1167. 